\title{
Nódulo Umbilical Metastático (Nódulo da Irmã Maria José): um Sinal de Alerta para Tumoração Maligna Intra-abdominal - Relato de Caso
}

\author{
Sister Mary Joseph's nodule: a Warning Sign for Intra-abdominal \\ Malignant Tumors. A Case Report
}

Homero Soares Fogaça ${ }^{1}$, Vera Lúcia Antunes Chagas ${ }^{2}$, Yolanda Faia Manhães Tolentino ${ }^{1}$, Vinicius Costa de Medeiros Ribeiro ${ }^{1}$, Lia Laura Lewes Ximenes ${ }^{3}$

\begin{abstract}
RESUMO
O nódulo da "Irmã Maria José" é tumor metastático que acomete a cicatriz umbilical e pode ser a primeira evidência de neoplasia intra-abdominal disseminada. Os autores relatam o caso de uma paciente com o nódulo da "Irmã Maria José" no qual o diagnóstico do tumor primário só foi possivel por meio dos testes imuno-histoquímicos da lesão biopsiada da pele.
\end{abstract}

PALAVRAS-CHAVE: Nódulo umbilical. Nódulo da Irmã Maria José. Metástase cutânea. Ovário: carcinoma.

\section{Introdução}

O primeiro relato de tumoração umbilical por câncer foi feito em 1846 por Walshe (Shetty) ${ }^{1}$ e o epônimo "Nódulo de Irmã Maria José" foi utilizado posteriormente para lesão metastática para cicatriz umbilical por Bailey². A Irmã Maria José, assistente que trabalhava no Hospital St Mary em Rochester, Minnesota, entre 1889 e 1939, observou a relação existente entre o nódulo umbilical e a presença de tumor maligno intra-abdominal ${ }^{1,3-5}$.

O Nódulo de "Irmã Maria José", pequeno nódulo endurecido na região umbilical, podendo ser proeminente ou não, é, às vezes, o primeiro ou único sinal de neoplasia maligna ${ }^{1,3,5-8}$. Os tumores que mais freqüentemente cursam com nódulo umbilical metastático se originam no trato gastrointestinal (estômago ${ }^{5}$, cólon ${ }^{6,9-11}$, pâncreas ${ }^{7}$ e intestino delgado ${ }^{12}$ ), no trato gênito-urinário (ová-

${ }^{1}$ Departamento de Medicina Interna, Serviço de Gastroenterologia, Hospital Universitário Clementino Fraga Filho, Universidade Federal do Rio de Janeiro, Rio de Janeiro, RJ

${ }^{2}$ Departamento de Patologia, Hospital Universitário Clementino Fraga Filho, Universidade Federal do Rio de Janeiro, Rio de Janeiro, RJ

${ }^{3}$ Instituto Oswaldo Cruz, Fundação Oswaldo Cruz, Rio de Janeiro, RJ.

Correspondência:

Homero Soares Fogaça

Av. Francisco Alves, 196 - I. Governador

21940-260 - Rio de Janeiro - RJ

e-mail: fogacas@openlink.com.br rio $^{13}$, endométrio ${ }^{14}$, colo uterino ${ }^{15} \mathrm{e}^{\operatorname{rim}^{16}}{ }^{16}$. Mais raramente podemos encontrar metástase umbilical decorrente de linfoma ${ }^{17,18}$, e câncer de mama ${ }^{19}$ e do tumor carcinóide ${ }^{20}$. A via de disseminação pode ser hematogênica, linfática ou por contigüidade $^{1,3,4}$. A avaliação histopatológica do nódulo pode ser feita por biópsia excisional ou aspiração por agulha ${ }^{1,3-5,13,21}$. A metástase cutânea para região umbilical freqüentemente está associada com rápida progressão da doença neoplásica, levando ao óbito em poucos meses, raramente com sobrevida excedendo $5 \operatorname{anos}^{1,3,7,12,13}$. No diagnóstico diferencial deve ser excluído hérnia umbilical, infecção local com granuloma piogênico e tumor primário maligno ou benigno da região umbilical.

\section{Relato do Caso}

Mulher, 69 anos, branca, com história de há aproximadamente 6 meses ter iniciado quadro de dor abdominal mal caracterizada, localizada em hipogástrio, aumento da circunferência abdominal, constipação intestinal e perda de $6 \mathrm{~kg}$ no período. Há 2 meses observou o aparecimento nodulação umbilical indolor. Procurou atendimento no ambulatório de Gastroenterologia, sendo internada em 20 de março de 1999 para investigação diagnóstica.

Ao exame físico apresentava-se em mau estado geral, emagrecida, com aumento do volu- 
me abdominal. À palpação do abdome foi evidenciada volumosa massa de limites imprecisos, consistência cística, indolor, que ocupava as regiões mesogástrica, hipogástrica e flanco esquerdo. $\mathrm{Na}$ cicatriz umbilical havia um nódulo de aproximadamente $1,0 \mathrm{~cm}$ de diâmetro, com consistência elástica e de coloração acastanhada.

No mesmo dia da internação, a paciente foi submetida à ultra-sonografia de abdome total sendo evidenciado ascite e massa cística ocupando o flanco esquerdo e hipogástrio. Foi imediatamente submetida à tomografia computadorizada contrastada (contraste oral e venoso) que mostrou também ascite e uma infiltração cutânea da região umbilical (Figura 1) que tinha solução de continuidade com a massa cística na região hipogástrica, sendo o aspecto sugestivo de tumor de ovário.

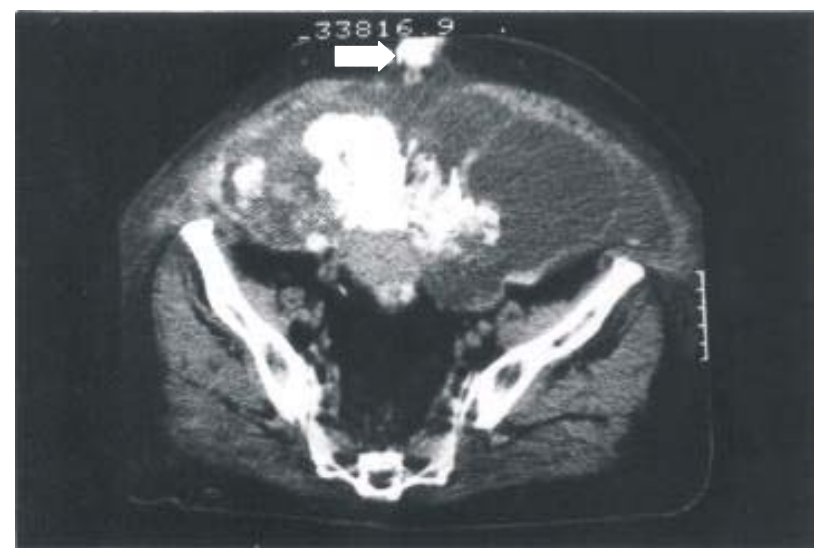

Figura 1 - Tomografia computadorizada abdominal demonstrando ascite e a cicatriz umbilical infiltrada (seta)

A paciente foi então submetida à biópsia excisional da lesão umbilical. A macroscopia do material de biópsia media $1 \mathrm{~cm}$ de diâmetro, com coloração acastanhada e consistência firme-elástica. Aos cortes evidenciavam-se numerosos cistos preenchidos por substância brancacenta homogênea. Este fragmento foi submetido à coloração com hematoxicilina-eosina e levado à análise imuno-histoquímica. O diagnóstico histopatológico foi de adenocarcinoma metastático, com provável origem em ovário. A neoplasia, que comprometia desde a hipoderme até o terço superior da derme, era representada por pequenos maciços celulares e por estruturas papilíferas com células pseudoestratificadas, de núcleos vesiculosos e pleomórficos, com nucléolos proeminentes e ocasionais mitoses, às quais associavam-se numerosos focos de calcificação freqüentemente de padrão psamomatoso (Figura 2A, 2B). A avaliação imunohistoquímica foi realizada utilizando-se a técnica de avidina-biotina-peroxidase com anticorpos monoclonais dirigidos ao $\mathrm{CA}-125$ e ao $\mathrm{CEA}^{22}$. A imunorreatividade para o CA-125 foi observada predominantemente na região apical e na superfície luminal das membranas celulares que compõem as projeções papilares (Figura 2C). Não houve imunorreatividade para o CEA.
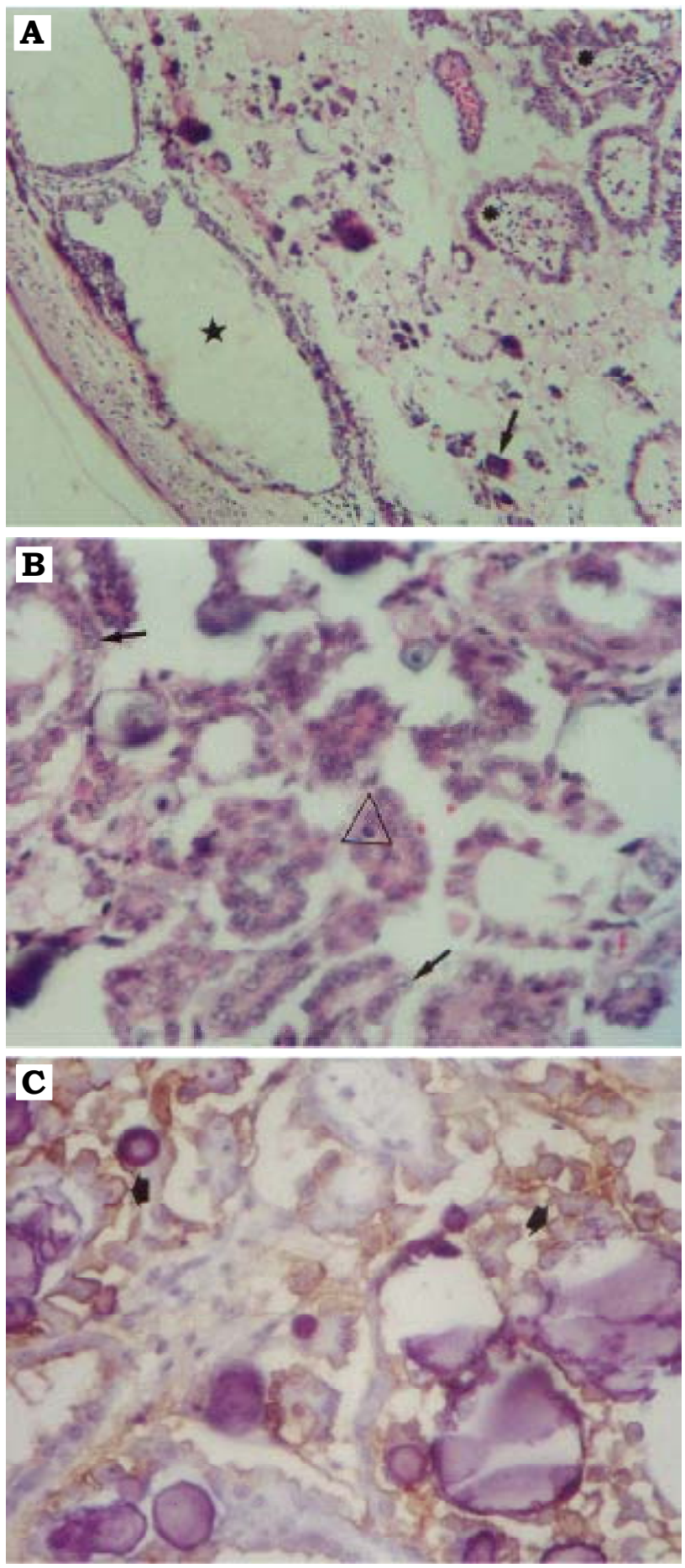

Figura 2 - Achados histopatológicos e imuno-histoquímicos na biópsia do nódulo umbilical.

A. Pele da área umbilical com implante de adenocarcinoma evidenciando cistos glandulares (estrela), papila (asterisco) e corpos psamomatosos (seta) H-E 100X $B$. Detalhe da figura A com células colunares e cubóides atípicas com núcleos grandes e nucléolos proeminentes (seta) com mitoses ocasionais (triângulo) H-E 400X.

C. Imunorreatividade para o CA-125 demonstrado nas células glandulares e nas papilas (seta) Imuno-histoquímica com fundo H-E 400X. 
A paciente veio a falecer cerca de 12 horas após a admissão e o diagnóstico de adenocarcinoma ovariano metastático foi retrospectivo, após ter sido liberado o laudo da análise imuno-histoquímica do nódulo umbilical.

\section{Discussão}

O nódulo da "Irmã Maria José" sugere a existência de neoplasia intra-abdominal disseminada ${ }^{1-}$ 5,23,24. Este nódulo é achado raro ao exame físico e pode ser o primeiro sinal de neoplasia intra-abdominal avançada e geralmente não mais passivel de ressecção. A observação do nódulo da "Irmã Maria José" deve ser considerada indicador de neoplasia intra-abdominal cuja origem pode ser principalmente o trato gastrointestinal (52\%) ou ginecológico (28\%). Em 40\% dos casos o nódulo pode significar recidiva de neoplasias previamente ressecadas $^{25}$. Os tumores que mais comumente cursam com este tipo de metástase cutânea são: ovário na mulher e estômago e cólon no homem ${ }^{1,4,5}$.

Ainda que possamos encontrar corpos psamomatosos na análise histopatológica de outros carcinomas papilares como, carcinoma papilar da tireóide e no carcinoma papilar de células renais, o adenocarcinoma de ovário foi sugerido como sendo a origem da metástase umbilical devido aos achados nos métodos de imagem com a observação de psamomas relacionados a projeções papiliferas na histologia. Corpos psamomatosos são freqüentemente encontrados no carcinoma de ovário seroso e caracterizam este tipo de tumor ${ }^{26}$, ocorrendo em intensidade variada tanto nos tumores limitrofes quanto nos francamente malignos ${ }^{27}$.

A hipótese de adenocarcinoma de ovário metastático foi reforçada pela positividade do anticorpo anti-CA-125, verificada à imunohistoquímica, nas células neoplásicas. A presença deste antígeno pode ser observada em $90 \%$ dos pacientes com adenocarcinoma de ovário, principalmente naqueles do tipo seroso ${ }^{20,28,29}$. Neste tipo de tumor ovariano existe importante correlação entre a positividade do teste imuno-histoquímico e os niveis séricos elevados de CA-125 $5^{30}$, que não conseguimos determinar em virtude do óbito ter ocorrido precocemente.

No caso relatado, o nódulo cutâneo umbilical metastático ("Nódulo da Irmã Maria José") e a positividade do anti-CA-125 no teste imunohistoquímico, juntamente com os métodos de imagem (ultra-sonografia e tomografia computadorizada) nos possibilitou, ainda que retrospectivamente, fazer o diagnóstico de adenocarcinoma de ovário metastático.
Enfatizamos a necessidade da avaliação criteriosa e cuidadosa de qualquer lesão umbilical e a sua correlação com o diagnóstico histopatológico. No caso de malignidade, o uso de técnicas de imuno-histoquímica pode auxiliar na definição da origem do sítio primário da neoplasia.

\section{ABSTRACT}

The umbilical metastatic tumoral nodule, Sister Mary Joseph's nodule, is a rare clinical sign indicative of disseminated intra-abdominal tumor, and may be the first evidence of malignancy. The authors report a case of a patient with umbilical metastatic nodule in whom the diagnosis of the primary tumor was possible by immunohistochemistry of the lesion biopsy.

KEYWORDS: Umbilical metastatic tumor. Sister Mary Joseph's nodule. Ovarian neoplasms.

\section{Agradecimentos}

Ivana Höfke e Antonia Mesquita pela assistência na técnica de imuno-histoquimica.

\section{Referências}

1. Shetty MR. Metastatic tumors of the umbilicus: a review 1830-1989. J Surg Oncol 1990; 45:212-5.

2. Bailey H. Semiologia quirúrgica. $13^{\mathrm{a}} \mathrm{ed}$. Barcelona: Toray; 1963. p.376-7.

3. Schneider V, Smyczek B. Sister Mary Joseph's nodule: diagnosis of umbilical metastases by fine needle aspiration. Acta Cytol 1990; 34:555-8.

4. Requena Caballero L, Vazquez Lopez F, Requena Caballero C, et al. Metastatic umbilical cancer : Sister Mary Joseph's nodule: report of two cases. J Dermatol Surg Oncol 1988; 14:664-7.

5. Samitz MH. Umbilical metastasis from carcinoma of the stomach. Sister Joseph's nodule. Arch Dermatol 1975; 111:1478-9.

6. Zeligman I, Schwilm A. Umbilical metastasis from carcinoma of the colon. Arch Dermatol 1974; 110:911-2.

7. Chatterjee SN, Bauer HM. Umbilical metastasis from carcinoma of the pancreas. Arch Dermatol 1980; 116:954-5.

8. Kort R, Fazaa B, Zermani R, Letawe C, Kamoun MR, Pierard GE. Sister Mary Joseph's nodule and inaugural cutaneous metastases of gastrointestinal carcinomas. Acta Clin Belg 1995; 50:25-7. 
9. Falchi M, Cecchini G, Derchi LE. Umbilical metastasis as first sign of cecal carcinoma in a cirrhotic patient (sister Mary Joseph nodule). Report of a case. Radiol Med (Torino) 1999; 98:94-6.

10.Barnes J, Patel V, Lee M. Adenocarcinoma of the colon presenting with a Sister Mary Joseph's nodule and Trousseau's syndrome. Cutis 1995; 56:270-2.

11.Khan AJ, Cook B. Metastatic carcinoma of umbilicus: Sister Mary Joseph's nodule. Cutis 1997; 60:297-8.

12.Powell FC, Cooper AJ, Massa MC, Goellner JR, Su WP. Leiomyosarcoma of the small intestine mestastatic to the umbilicus. Arch Dermatol 1984; 120:402-3.

13. Heatley MK, Toner PG. Sister Mary Joseph's nodule: a study of the incidence of biopsied umbilical secondary tumors in a defined population. Br J Surg 1989; 76:728-9.

14.Patel KS, Watkins RM. Recurrent endometrial adenocarcinoma presenting as an umbilical metastasis (Sister Mary Joseph's nodule). Br J Clin Pract 1992; 46:69-70.

15. Edozien LC, Obed JY, Williams GA, Ladipo OA. Squamous carcinoma of the cervix metastatic to the umbilicus. A case report. Eur J Gynaecol Oncol 1994; 15:152-3.

16. Chen P, Middlebrook MR, Goldman SM, Sandler CM. Sister Mary Joseph's nodule from metastatic renal cell carcinoma. J Comput Assist Tomogr 1998; 22:756-7.

17.Chagpar A, Carter JW. Lymphoma presenting as a Sister Mary Joseph's nodule. Am Surg 1998; 64:799800.

18.Dornier C, Reichert-Penetrat S, Barbaud A, Kaise W, Schmutz JL. Lymphoma presenting as Sister Mary Joseph's nodule. Ann Dermatol Venereol 2000; 127:732-4.

19.de la Cruz Mera A, Vargas-Castrillon J, de Agustin P, Perez-Barrios A. Fine needle aspiration biopsy diagnosis of Sister Mary Joseph's nodule 13 years after mastectomy. Acta Cytol 1991; 35:790.
20.Brody HJ, Stallings WP, Fine RM, Someren A. Carcinoid in an umbilical nodule. Arch Dermatol 1978; 114:570-2.

21.Fleming MV, Oertel YC. Eight cases of Sister Mary Joseph's nodule diagnosed by fine-needle aspiration. Diagn Cytopathol 1993; 9:32-6.

22.Hsu SM, Raine L, Fanger H. The use of antiavidin antibody and avidin-biotin-peroxidase complex in immunoperoxidase technics. Am J Clin Pathol 1981; 75:816-21.

23.Touraud JP, Lentz N, Dutronc Y, Mercier E, Sagot $\mathrm{P}$, Lambert D. Umbilical cutaneous metastasis (or Sister Mary Joseph's nodule) disclosing ovarian adenocarcinoma. Gynecol Obstet Fertil 2000; 28:719-21.

24.Amaro R, Goldstein JA, Cely CM, Rogers AI. Pseudo Sister Mary Joseph's nodule. Am J Gastroenterol 1999; 94:1949-50.

25.Giner Galvan V. Sister Mary Joseph's nodule: its clinical significance and management. An Med Interna 1999; 16:365-70.

26. Cotran RS, Kumar V, Robbins SL. Robbins pathologic basis of disease. $6^{\text {th }}$ ed. Philadelphia: WB Saunders; 1999. p.260-327.

27.Sternberg S. Diagnostic surgical pathology. $2^{\text {nd }} e d$. New York: Raven Press; 1994. p.73-4.

28.van Niekerk CC, Boermann OC, Ramaekers FC, Poels LG. Marker profile of different phases in the transition of normal human ovarian epithelium to ovarian carcinomas. Am J Pathol 1991; 138:455-63.

29.Nolan LP, Heatley MK. The value of immunocystochemistry in distinguishing between clear cell carcinoma of the kidney and ovary. Int $\mathrm{J}$ Gynecol Pathol 2001; 20:155-9.

30.Leake J, Woolas RP, Daniel J, Oram DH, Brown CL. Immunocytochemical and serological expression of CA 125: A clinicopathological study of 40 malignant ovarian epithelial tumours. Histopathology 1994; 24:57-64.

Recebido em: 8/ 1/2003 Aceito com modificações em: 2/6/2003 